\title{
Ab welchem Wert muss an Krebs gedacht werden?
}

Frage: Wie ist bei einem 60-jährigen langjährigen Raucher mit chronischer Bronchitis und unauffälligem Röntgen-Thorax ein NSE(neuronenspezifische Enolase)-Wert von 20,4 $\mu \mathrm{g} / \mathrm{l}$ (in meinem Labor Norm bis 18,3) zu bewerten? Würden Sie eine weitere Diagnostik bezüglich eines Bronchialkarzinoms für notwendig erachten?

Dr. Eckehard Meissner: Im Gegensatz zum Tumormarker CEA (Carcinoembryonales Antigen) gib es für die NSE keinen sicher nachgewiesenen Zusammenhang mit dem Rauchen, d. h. erhöhte Werte bei Rauchern. NSE-Werte $>25 \mu \mathrm{g} / \mathrm{l}$ sprechen mit hoher Wahrscheinlichkeit für ein kleinzelliges Bronchialkarzinom (SCLC).

Der von Ihnen mitgeteilte Wert liegt damit in der Grauzone. Vor der Diagnostik hinsichtlich eines BC sollte der Wert hinsichtlich der Umstände bei der Abnahme einmal bewertet werden. Es gibt bei der NSE-Bestimmung leider Störfaktoren, die zu falsch-positiven Werten führen können. Hier sind besonders die zu lange Lagerung des Blutes vor dem Abseren bzw. die Einsendung von Vollblut ins Labor zu nennen (Zeitintervall zwischen Blutabnahme und Zentrifugation des Blutes max. 60, besser nur $30 \mathrm{Mi}-$ nuten. Danach kommt es durch Zelllyse zur NSE-Freisetzung aus Thrombozyten und Erythrozyten).

Expertenrat Pneumologie,

www.springermedizin.de

„Herr Doktor, ich vertrage Erdbeeren nicht!"

\section{Echte Allergie oder nur Unverträglichkeit - wie abklären?}

Frage: Immer wieder habe ich Patienten, die angeben, bestimmte Lebensmittel (z.B. Milch/Erdbeeren) "nicht zu vertragen“. Wie stelle ich am schnellsten fest, ob eine echte Nahrungsmittelallergie vorliegt? Was ist von sublingualen oder intradermalen Provokationstests zu halten?

Prof. H. S. FüeßI, Haar: Man unterscheidet zwischen immunologisch bedingten Nahrungsmittelallergien und $\mathrm{Nah}$ rungsmittelintoleranzen ohne immunologische Beteiligung. IgG-vermittelte Nahrungsmittelallergien beruhen auf Proteinallergien und können mit wissenschaftlich evaluierten Tests überprüft werden. Dazu gehören neben der allergologischen Anamnese Prick-, Scratch- und Reibetests, die Bestimmung spezifischer IgG und Provokationstests. Für Nahrungsmittelintoleranzen stehen keine Allergietests zur Verfügung, so dass die Diagnose ausschließlich auf Anamnese, Führung eines Symptomentagebuchs, Eliminationsdiäten und kontrollierten Provokationen beruht. Weitere Informatio- nen findet man unter www.dgaki.de und www.awmf-online.de oder im Springer Medizin Schwerpunkt „Allergologie“.

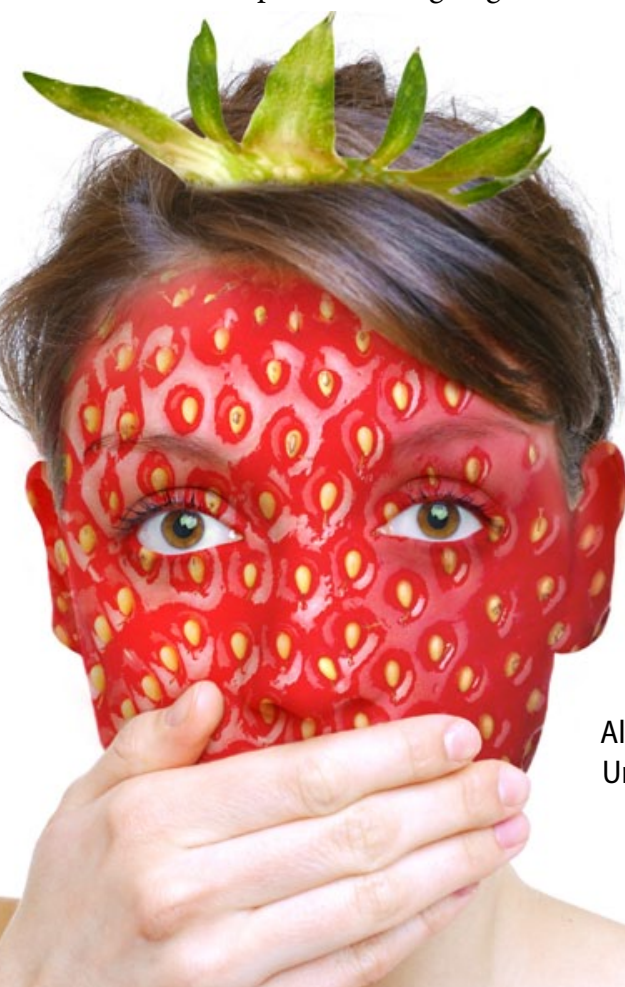

Dr. Peter Stiefelhagen, Hachenburg: Nicht jede Nahrungsmittelunverträglichkeit ist eine Allergie. Zunächst sollte immer eine Maldigestion durch Enzymmangel wie bei einer Laktose- oder Fruktoseintoleranz mittels Probemahlzeit mit Bestimmung des Blutzuckers oder besser mittel $\mathrm{H}_{2}$-Atemtest nachgewiesen oder ausgeschlossen werden. Eindeutig für eine Allergie spricht, wenn neben Magen-Darm-Beschwerden, Reaktionen an anderen Schleimhäuten (Rhinitis oder Konjunktivitis) oder der Haut (Urtikaria) oder sogar ein anaphylaktischer Schock aufgetreten sind. Die sublinguale Testung ist noch nicht so etabliert.

Expertenrat Allgemeinmedizin,

www.springermedizin.de

Allergisch? Oder nur eine Unverträglickeit? 